\title{
ENCEPHALITIC LISTERIOSIS IN SHEEP AT KAFRELSHEIKH GOVERNORATE; DIAGNOSTIC AND THERAPEUTIC STUDY
}

\author{
Hamdy abou Sheasha, Yamen, M. Hegazy, Atef F. Oreiby, \\ Amin M Thahoun, Magdy H. Al-Gaabary \\ Animal Medicine Department, Faculty of Veterinary Medicine, \\ Kafrelsheikh University (Egypt).
}

\begin{abstract}
Encephalitic listeriosis in sheep has been investigated in Kafrelsheikh governorate to confirm its existence as well as evaluation of some treatment trials. In this study, 1940 sheep were examined between November, 2013 and October, 2014. The clinical signs were ranged from a mild, stage I, with good prognosis to severe terminating by death, stage III, exhibiting flaccid paralysis, hemiplagia and tetraplagia. Listeriosis was confirmed by bacterial isolation and identification of the causative agent, histopathological examination and PCR. Treatment trials showed that early intervention with prolonged course of antimicrobial which pass the blood-brain barrier was successful in curing the diseased animals except those in stage III of the disease. Also, it is important to use ancillary treatment which composed of vitamin $B$ complex and anti-inflammatory, besides changing feeding regime.
\end{abstract}

\section{INTRODUCTION}

Sheep, as productive animals, have an important value as it is a valuable source of meat, milk, leather. Besides its short production cycle, sheep could feed on residues of the crops (El-Sukhon and Nareman 2004). Furthermore, sheep have a religious importance in Islamic countries: it is mentioned in the Quran and usually it is slaughtered in Eid al-Adha. 
Sheep are affected by many bacterial diseases. One of such diseases is listeriosis. Listeriosis has negative effects on sheep production; septicemic listeriosis is fatal mainly in lambs, encephalitic form may be ended by death and many cases of abortion in ewes due to reproductive form (Fthenakis et al. 1998). Listeriosis has a zoonotic importance where listeria monocytogenes is a major human bacterial food born pathogen that induces meningitis, encephalitis, sepsis, fetal death and pre mature birth (Irene et al. 2002).

Clinically, listeriosis in sheep exhibits several forms: encephalitis, septicemia, abortion and mastitis. Encephalitis is a characteristic form of ovine listeriosis which is usually termed "circling disease". Early signs of circling disease are fever, separation from the flock, depression, circling, in coordination and easily fall down. The signs progress rapidly due to affection of central nervous system and unilateral or bilateral cranial nerves damage. The course of the disease is usually 2-3 weeks and mainly ended by death (Roberts and Wiedmann 2003).

Sheep usually get infection with Listeria monocytogenes through the ingestion of contaminated ration. There is a direct link between silage feeding and listeriosis, mainly bad quality silage which poor fermented and badly preserved

Different diagnostic methods are being used to identify ovine Listeriosis. Isolation of L. monocytogenes and histopathogical examination are the classical methods used for laboratory diagnosis of listeriosis. Isolation may be unsuccessful even when samples are positive. Culture - negative cases can be a result of few or no bacteria in the lesion especially in treated cases. Neural listeriosis requires cold enrichment to recover the organism then subculture on sheep blood agar 
and palcam agar. Using histopathological method on brain stem, pons and cerebellum showed perivascular cuffing, micro abscesses, focal necrosis and neutrophelia (Campero et al 2002). The combined use of PCR detection of listeria and anti-LLO IgG immunoassay (ELISA) by means of commercial kits, allow a rapid and effective detection of $L$. monocytogenes infection in early stage, before seroconversion and in a later stage even after antibiotic therapy (Amagliani et al. 2006).

The recovery rate is higher if the treatment is administered early in the course of the disease. Treatment of severely ill, recumbent animals and cases of spinal myelitis are poorly responsive to treatment.

There are no previous studies on meningeoencephalitic listeriosis in sheep in Kafrelsheikh Governorate, Egypt, although many clinical cases and vigorous out breaks of sheep suffering from meningeoencephalitis were observed which named among shepherds 'Doshan'. Also, there is a huge debate on the causes of Doshan either is it cenurosis or encephalitic listeriosis. So the study is aimed to investigate the causes of Doshan at Kafrelsheikh governorate with different diagnostic methods. Also, to study the effect of different regimes of treatment and control of listeriosis in sheep at Kafrelsheikh governorate.

\section{MATERIALS AND METHODS}

\section{1- Animals and clinical examination:}

A total of 1940 sheep of 5 different flocks from different localities at Kafrelsheikh Governorate were examined for encephalitic listeriosis. The clinical examination was carried out with special reference to nervous signs according to Kelly 1990. This study was carried out throughout one year from November 2013 to October 2014. 


\section{2- Samples:}

Brains of recently dead sheep as well as slaughtered ill ones were aseptically collected. Each sample was divided into two parts; one part for bacteriological procedures and the other part were preserved in formalin $10 \%$ for histopathological examination.

\section{3- Histopathological examination:}

Brains specimens including Pons, brain stem, medulla oblongata and cerebellum had been collected. The specimens were put in formalin $10 \%$. After fixation the specimens were processed by paraffin embedding techniques and stained by hematoxylin and eosin for routine histopathological examination (Harris 1998).

4- Bacteriological isolation and identification according to (Quinn et al. 1999):

\section{a. Enrichment procedures:}

Twenty-five grams from each brain sample were blended with a moulinex type blender equipped with metallic flask with $225 \mathrm{ml}$ phosphate peptone broth. The samples in the primary enrichment broth were incubated at $30 \mathrm{c}$ for $24 \mathrm{~h}$, after that $0.1 \mathrm{ml}$ of inoculated phosphate peptone broth was transferred to $10 \mathrm{ml}$ listeria enrichment broth and incubated at $30 \mathrm{c}$ for 24 hours (McClain and lee 1988).

\section{b. Isolation and identification procedure:-}

- A loopful of incubated enrichment broth was streaked on blood agar to record beta hemolysis (Curtis et al 1989). A loopful from pure colonies was sub cultured into a placam agar plate, which was then incubated at $30 \mathrm{c}$ for 24-48 hr (Van-Netten et al., 1989). Pure isolates were identified according to Anneschuchat et al. (1991) and

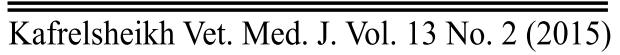


Donnelly (1992). Films were made from previously prepared pure cultures. The films were stained with Gram's stains and examined microscopically for non sporulated gram positive coccobacilli occurring in short chains of three to five organisms with a typical diphtheroid palisade arrangement.

- PCR Identification

Ten isolates suspected to be Listeria monocytogenes were used to be typed by PCR. PCR steps were DNA Extraction, PCR amplification, PCR Cycling and Agarose gel electrophoresis according to (Borucki et al., 2003). Positive bands were expected at $800 \mathrm{bp}$. The primers have the following characters and sequences according to (Dongyou et al 2007) as shown in table 1 below.

Table (1): Primer sequence of Listeria monocytogenes

\begin{tabular}{|c||c||c||c|c|c||}
\hline Reference & $\begin{array}{c}\text { Primer } \\
\text { size }\end{array}$ & $\begin{array}{c}\text { Number } \\
\text { of bases }\end{array}$ & Sequence & $\begin{array}{c}\text { Target } \\
\text { gene }\end{array}$ & $\begin{array}{c}\text { Name of } \\
\text { primer }\end{array}$ \\
\hline \hline \multirow{2}{*}{$\begin{array}{c}\text { Dongyou et al } \\
\mathbf{2 0 0 7}\end{array}$} & $800 \mathrm{bp}$ & 20 & ACG AGT AAC GGG ACA AAT GC-3 & inlA For & Forward \\
\cline { 2 - 6 } & $800 \mathrm{bp}$ & 20 & CCC GAC AGT GGT GCT AGA TT-3 & inlA Rev & Reverse \\
\cline { 2 - 6 }
\end{tabular}

\section{5- Treatment trial}

Treatment of diseased animals was conducted by using antibiotics that have high concentration in brain and passing blood brain barrier. In order to follow the best choice for treatment we divide the animals into 3 groups according to type of antimicrobial used in treatment each group as follows:

Group (1): Animals of this group were 46 sheep that received Pencillin G Sodium (Memphis C. I. D. CO) was used by the dose of 44000 I.U for $\mathrm{kg} \mathrm{BW}$ for one week. 
Group (2): Animals of this group were 68 sheep that received Ampicillin (Chemical industries development Co) (CID) BNO.1107111) was used in a dose of $20 \mathrm{mg} / \mathrm{Kg}$ BWT twice daily for one week intravenous $(1 / \mathrm{V})$.

Group (3): Animals of this group were 38 sheep that received Borgal injection (sulfa doxin and tri-methoprim) was used $3 \mathrm{ml} / 50 \mathrm{~kg}$ BWT daily for one week (Schering plough Germany).

Vitamins B complex was also used to all treated animals for regeneration of nervous tissue. The anti-inflammatory drugs also were used by large doses. The most important was mainly dexamethasone for non-pregnant to reduce brain edema. All animals were observed till complete recovery or death.

\section{RESULTS}

\section{A. Clinical signs:}

A total of 152 animals had clinical signs of encephalitic listeriosis. Sheep were suffering from varied degrees of nervous manifestations. The recorded signs of encephalitic form of listeriosis were classified according to the severity into:

Stage I This stage was characterized by mild symptoms and good prognosis. The affected animals showed fever, depression, loss of menaces, and mild deviation of neck. The affected animals also showed head pressing against hard objects, hanging saliva from mouth, drooping of ear and drooping eye lid (Fig. 1). 
Stage II or moderate stage. During this stage animals were more affected than that of stage I. They showed moderate signs and fair prognosis. In this stage the signs were characterized by asymmetric closure of the jaw, difficult in swallowing, paresis and paralysis of the tongue. The animal also showed nystigmus, compulsive circling sever twisting head. At the end of this stage the animals showed loss of menace, and were easy fall dawn and unable to eat (Fig 2).

Stage III or severe stage: during This stage the signs were severe with bad prognosis at the end the ill animal became lateral recumbent, unable to rise, showing convulsion,, paddling movement, animal completely anorectic and died (Fig 3).

\section{B. Result of bacteriological examinations:}

The obtained colonies small transparent and of narrow zone of beta haemolysis on blood agar (Fig. 4). Microscopically sproulated Gram positive coccobacilli had been illustrated in (Fig. 5). PCR products of (800bp) were obtained. This product was at the expected band size indicating the identity Listeria monocytogenes (Fig. 6).

\section{Histopathological findings:}

The examined sections showed edema, hemorrhage, micro abscesses and accumulation of inflammatory cells lymphocytes, macrophages, gaint cells, neutrophils, plasma cells and histocytes around or in close to blood vessels forming perivascular cuffing and vasculitis (Fig 7 and 8). However, interestingly the micro abscesses were present mainly in brain stem between the midbrain and cervical part of spinal cord. These micro-abscesses were either single or multiple with necrotic and liquifactive changes with granuloma and malacia. Mono nuclear cells were located either in gray and or white matter. 


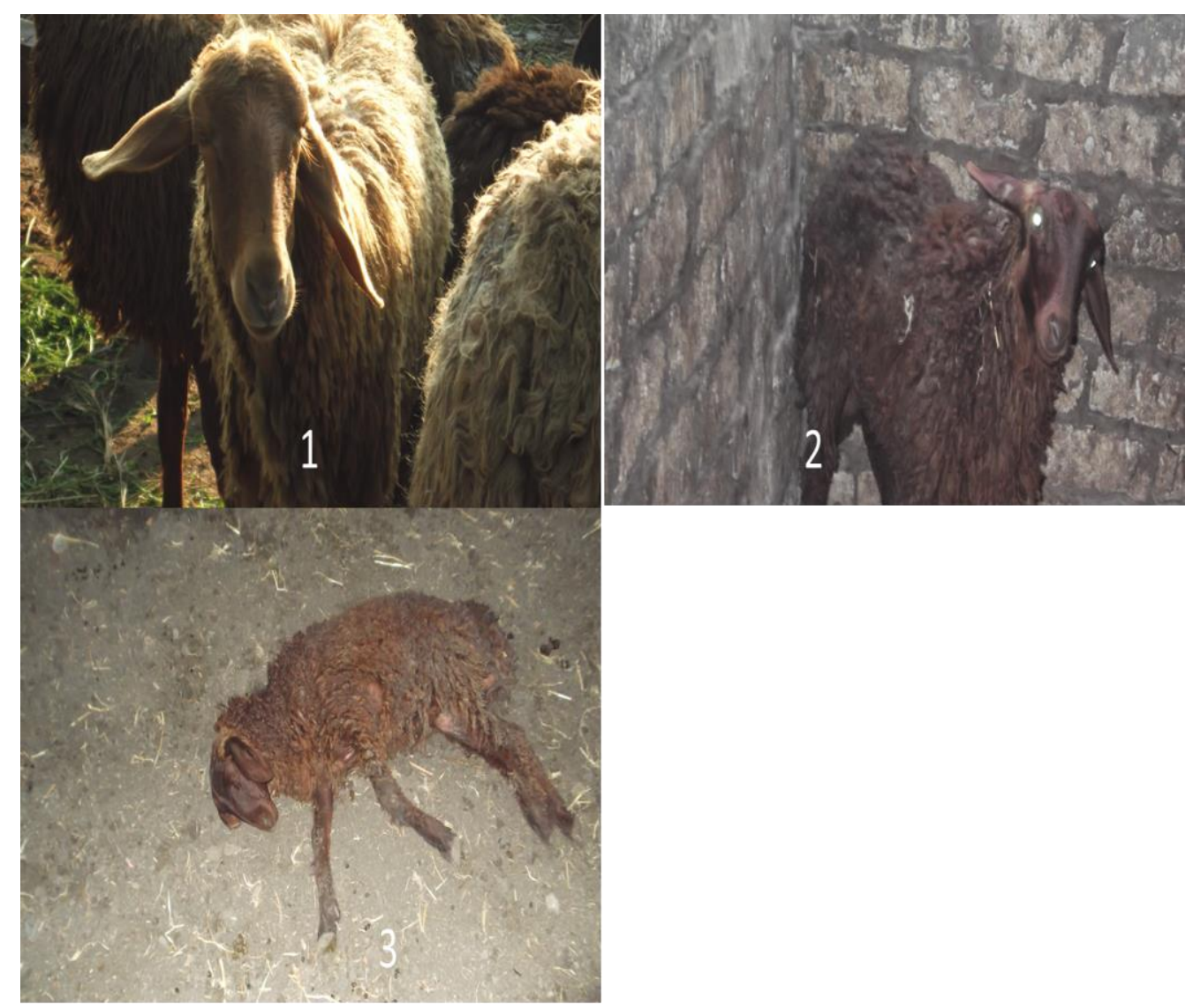

Fig. (1): showed ewe with dropped ear (Encephalitic listeriosis stage I)

Fig. (2): ewe showed nystigmus and severe twisting of head (Encephalitic listeriosis stage II )

Fig. (3): sheep suffered from terminal stage of listeriosis with lateral recumbence, Para paralysis and paddling movement (Encephalitic listeriosis stage III) . 


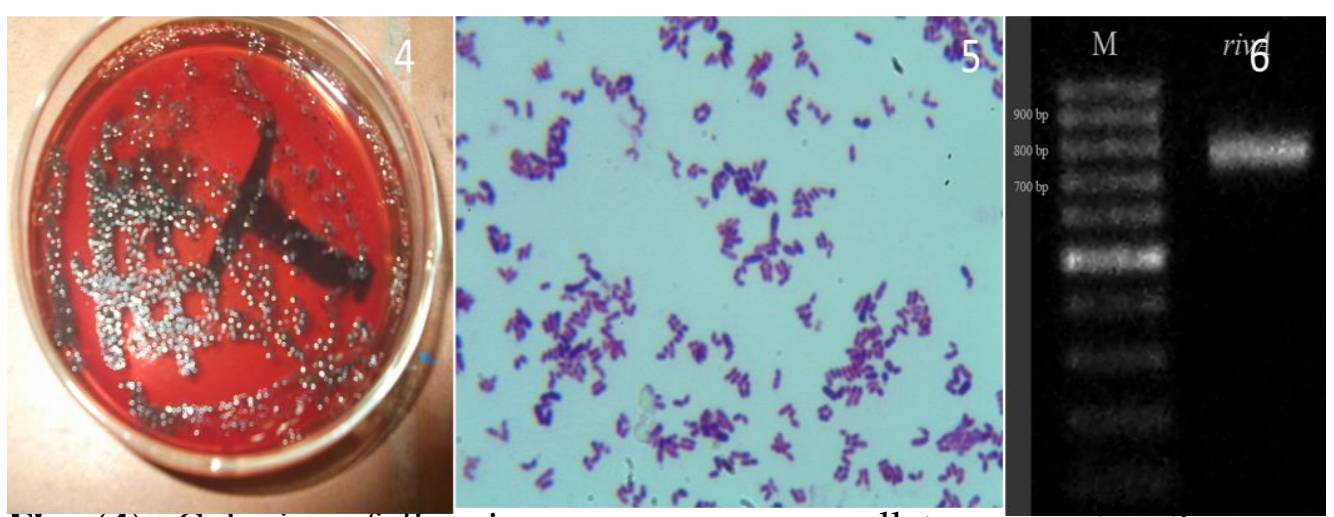

Fig. (4): Colonies of listeria monocytogenes small transparent with narrow zone of beta hemolysis on sheep blood agar.

Fig. (5): Gram positive coccobacilli of listeria monocytogenes

Fig. (6): positive result of P.C.R. of L. monocytogenes amplification product size at $800 \mathrm{bp}$.

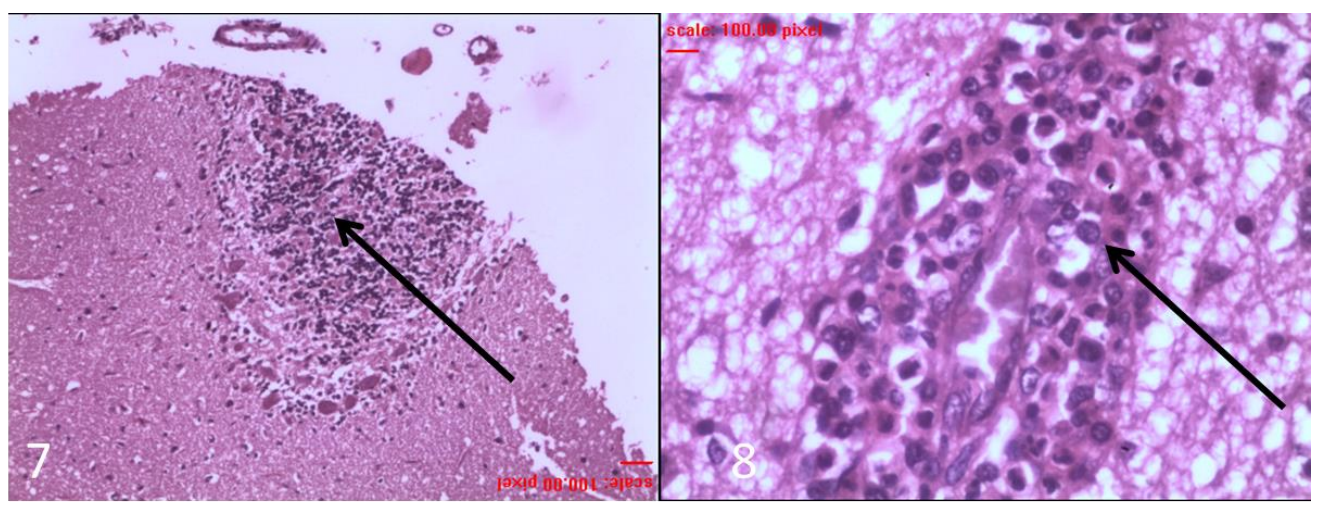

Fig. (7): Section in Pons of sheep brain showing micro abscess and liquifactive necrosis. Micro abscess represented by infiltration of lymphocytes, neutrophil and giant cell.

Fig. (8): section cerebral cortex showing cuffing and neutrophil infilteration. 


\section{Results of treatment trial}

Results of treatment trial showed that the recovery rate was $65.2 \%$ among animals in Group (A) which treated by penicillin $G$ where 30 recovered from 46. In group (B) treated by Ampicillin. The number of recovered cases were 32 from 68and its percentage were 47.1\%.In group (C) treated by borgal. The number of recovered cases were 29 from 38 and its percentage were $76.3 \%$ (Table 2).The recovery rate among animals of groups $\mathrm{A}$ and $\mathrm{C}$ were significantly higher than in animals in group B at $P<0.05$.

The case fatality and treatment failure was significantly higher in animals in stage III than those animals of stage I and II at $\mathrm{P}<0.05$. (Table 3)

The case fatality and treatment failure was significantly higher in young animals of less than 6 months age than those older animals at $\mathrm{P}<$ 0.05. (Table 4). The treatment efficacy in adult animals of more than 18 months age was the highest.

Table (3): Results of treatment trials among sheep using different antibiotics against Listeriosis encephalitis.

\begin{tabular}{|c|c|c|c|c|c|}
\hline Treatment group & Number of diseased & Number treated & $\%$ & Deaths & $\%$ \\
\hline A & 46 & 30 & 65.2 & 16 & 34.8 \\
\hline B & 68 & 32 & 47.1 & 36 & 52.9 \\
\hline $\mathrm{C}$ & 38 & 29 & 76.3 & 9 & 23.7 \\
\hline
\end{tabular}

Table (4): Recovery rate in relation to stage of the disease.

\begin{tabular}{|c|c|c|c|c|c|}
\hline Disease stage & Number of diseased & Number treated & $\%$ & Deaths & $\%$ \\
\hline Stage I & 42 & 34 & 81 & 8 & 19 \\
\hline Stage II & 89 & 46 & 51.7 & 43 & 48.3 \\
\hline Stage III & 21 & 1 & 4.8 & 20 & 95.6 \\
\hline
\end{tabular}

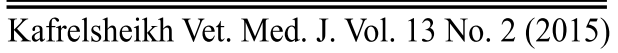


Encephalitic Listeriosis In Sheep At Kafrelsheikh ...

Table (5): Recovery rate in relation to age of treated animals.

\begin{tabular}{|c|c|c|c|c|c|}
\hline Age group & Number of diseased & Number treated & $\%$ & Deaths & $\%$ \\
\hline$<6$ month & 40 & 7 & 17.5 & 33 & 82.5 \\
\hline 6-18 month & 92 & 61 & 66.3 & 31 & 33.7 \\
\hline$>18$ month & 20 & 13 & 65 & 7 & 35 \\
\hline
\end{tabular}

\section{DISCUSSION}

Listeriosis constitutes a significant important disease to sheep production and human health. It causes severe economic losses due to deaths in sheep caused by encephalitic, septicemic and abortion forms in addition to the huge cost of medication and veterinary care .The human health concern is due to its zoonotic potential to cause meningitis, encephalitis, sepsis and fetal and premature death. In this work we investigate the causes of circling disease with different diagnostic methods. This to remove the obscureness on one of the most important signs in sheep flocks in Kafrelsheikh governorate (Doshan). Our research aimed to examine the effect of different regimes of treatment to select the best choice to be used in the treatment and control as well.

In this study, we examined 1940 animals in five different flocks for encephalitic listeriosis.

The clinical signs in the diseased sheep were classified according to the severity. It was divided into 3 groups according to the severity of the signs. The first group named stage Ithat group had head pressing against hard objects, hanging of saliva from mouth, drooping of ear and keratitis. Animals in This stage have good prognosis. The second group were in stage III stage that were characterized by more severe signs and fair prognosis. The signs in this stage were nystigmus, compulsive circling, severe twisting of the head and asymmetrical closure of the jaw. The severe or stage II showed flaccid paralysis. Para-paresis, or hemiparesis,

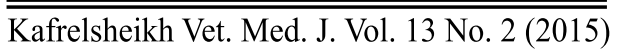


tetraplegia, complete paralysis in advanced cases and the animal became recumbent and unable to rise up and laying its neck toward the flank . This stage had a bad prognosis Resultes agree with. Fthenakis et al. (1998), Morin (2004) and Kumar et al., (2007).

Our results indicated that $28 \%$ of diseased sheep were in stage I. While as the stage II encephalitic listeriosis was about $58 \%$ of cases. Furthermore, the percentage of sheep showed stageIII was 14\%. These signs are not always gradual in nature; so we expect to see animals develop the moderate and severe forms without showing milder signs earlier. This is may be attributed to the affected part of the nervous tissue Low (2000), Braun et al.,( 2002), Ligios et al. (2004) and Brugere (2008). The majority of animals showed signs of stageII and this may reflect that one of the most affected part of the nervous tissue is mainly is the CN VIII. This nerve is responsible for hearing equilibrium sensation and this reflects the associated clinical signs of circling, ataxia and in equilibrium. This findings need further investigation on the reasons of the high risk of affection of this nerve and if it is related to the mode of infection of the high affinity of pathogen to this nerve.

Following on the previous researches we found that the diagnosis of encephalitic listeriosis encephalitis is very hard due to the lack of serological methods. The microbiological isolation methods also are less sensitive and difficult as it needs multiable cultivation on different media for 12 week. All these difficulties lead to underestimation of the encephalitic listeriosis in the examined flocks. It also gives false indications of the prevalence of listeriosis encephalitis. Therefore in this work we investigate the combination of epidemiological, chemical, microbiological, histo-pathological and PCR methods for diagosis of encephalitic listeriosis. 
Results of bacterial isolation showed that the obtained colonies were typically small transparent and of narrow zone of beta hemolysis. The colonies that were grown on Palcam agar were smooth, grayish color with raised surface like a button this as typically that of Listeria. Microscopical examination of Gram stained slides form specific colonies we noticed Gram positive cocco bacilli indicating possibility of being Listeria monocytogenes (Campero et al 2002, Thanna. 2002, Morin. 2004, Kumer et al. 2007).

Following a recent work that identifies Listeria monoctogenes by PCR via amplification of its specific gene inlA a known marker for Listeria monocytogenes. We successfully amplified this gene from our isolate using primers according to Dongyou et al. (2007). We obtained PCR products of $(800 \mathrm{bp})$. This product was at the expected band size indicating its identity as listeria monocytogenes (El-Sawalhy et al. 1999, Scott. 2004, Burgere. 2008) Both macroscopic and microscopic examination of brains from diseased animals showed edema hemorrhage, micro abscesses and accumulation of inflammatory cells lymphocytes, macrophages, giant cells, neutrophils, plasma cells and histocytes around or in close to blood vessels forming perivascular cuffing and vasculitis. However, interestingly the micro abscesses were present mainly in brain stem between the midbrain and cervical part of spinal cord. These microabscesses were either single or multiple with necrotic and liquifactive changes with granuloma and malacia. Mono nuclear cells were located either in gray and or white matter. Together these findings provide evidence and confirming for encephalitic Listeriosis identification (Schneider 1994, El sawalhy et al. 1999, Campero et al. 2002, Ligios et al. 2004). 
Diagnosis of this disease in our study highlights the importance of listeriosis as one of the main causes of circling in sheep flocks at the study area. So, It is advised for veterinarians who found similar clinical, epidemiological and post-mortem to expect listeriosis firstly. Confirmation of their diagnosis can be easily achievable through PCR for brain of one or more of died animals. This will save the time for isolation and it is highly sensitive and specific and gives the chance for early intervention.

The results of treatment of encephalitic listeriosis confirmed that its treatment was depending on prolonged course of antibiotics. We also set out to test a group of antibiotics that could have high concentration in brain following crossing the blood brain barrier. Taken together the results suggest that the best outcome of the disease treatment need early intervention by prolonged massive doses of antibiotic that could have high concentration in brain mainly (Sulfonamides and tri methoprim, Ampecillin and Pencillin G), plus strong anti-inflammatory mainly dexamethasone by double dose for non-pregnant sheep, supportive treatment by vitamin B complex and fluid therapy mainly manitol to decrease brain inflammation and good nursing to diseased animal.

Animals develop stage III were found seldom to respond to any type of treatment and the case fatality was significantly higher than other 2 stages. This is may be attributed to the massive damage of brain tissues which could not be regenerated with the used medications.

The treatment efficacy was found significantly higher among animals of ages more than 6 months age than young animals which suffered the highest case fatality rate. This is may be due to the lack of immunity of young animals Low and Renton (1985), Scott (1993) and Brugere (2008). 
Finally, we can conclude that what is named doushan is encephalitic form of listeriosis .The accurate diagnosis and early proper therapeutic intervention can result in high rate of recovery particularly in adult animals.

\section{REFERENCES}

- Amagliani A. B. C; Giammarini C. E.; Omiccioli A. D.; Merati A. G; Pezzotti D. G.; Filippini D. G.; Brandi A. B. and Magnani M. (2006): A combination of diagnostic tools for rapid screening of ovine listeriosis. Research in Veterinary Science 81: 185-189

- Anneschuchat; Bala,S.T. and Broome, C.V.(1991): Epidemiology of human listeriosis .Clin. Microb. Rev. April, 169-183.

- Borucki, WE. K., M. J. Krug, W. T. Mtiraoka, and D. R. Call. (2003): Discrimination among Listeria monocytogenes isolates using a mixed genome DNA microarray. Vet. Microbiol. 92:35 1-362.

- Braun. U.;Stehle. C. and Ehren Sperger, F. (2002): Clinical findings and treatment of listeriosis in 67 sheep and goats. Vet. Rec. 150: $38-42$

- Brugere, S. Picoiixa (2008): Ovine Listeriosis. Small Rumin. Research .76 (1-2): $12-20$

- Campero, C. M.; Odeon A. C.; Cipolla A. L.; Moore D. P.; Poso M. A., and Odriozola E.,(2002): Demonstration of Listeria monocytogenes by immunohistochemistry in formalin-fixed brain tissues from natural cases of ovine and bovine encephalitis. J. Vet. Med. B Infect. Dis. Vet. Public Health 49, 379-383. Carter, G.R.; Chengappa, M.M.; Roberts, A.W.; Williarn,G. and Rikihisa, X. (1995): Listeria in : Essentials of veterinary microbiology 51 Edin. (William and Wilkins) Pp 127-130. 
- Curtis, G.D.W.; Mitchell, R.G.; King, A.F. and Emma, J. (1989): A selective differential media for isolation of Listeria monocytogenes. App. Microb.8, 95-98.

- Dongyou. B (2007): Prenatal Death In Domestic Animals: The $20^{\text {th }}$ Symposium of the Nordic Committee for Veterinary Scientific Cooperation (NKVet) Reykjavik, Iceland. 26-27 April 2007

- Donnelly, C.W. (1992): Listeria. In compendium of methods for microbilogical examination of foods, 3rd Ecir, (Editors: Vanderzant, C. and Splitt sttoesser, D.F.) Pp 637-663.

- El-Sawalhy A. A.; Fouda T. A. And El-Sheib, A. F. (1999): An outbreak of ovine Listerial Meningeoencephalitis in Dakahlia Province. Zag. Vet. J. 27(5): 122 -134

- EI-Sukhon, S. N. and Nareman, M. Abu-Kuhail (2004): The association of pasteurella species and oestrus ovis in frontal sinuses in Awassi sheep in northern Jordan. Alex. Vet. Sci. (21): 1; 151 - 160

- Fthenakis, G.C., Saratsis, Ph., Tzora, A., Linde, K., (1998): Naturally occurring subclinical ovine mastitis associated with Listreia monocytogenes. Small Ruminant Research 31, 23-2 7.

- Harris, H.E. (1998): Veterinary pathology. Cited by Canton, Dury, Wallington and Cameron, 4 ed. Oxford, unipress, New York, Torento.

- Irene V. Wesley; David J. Larson; Karen M. Harmon; John B. Luchansky; Ann Ramos Schwartz (2002): A case report of sporadic ovine listerial menigoencephalitis in Iowa with an overview of livestock and human cases J. Vet. Diagn. Invest. 14:314-321

- Kelly, W. R. (1990): Veterinary clinical diagnosis. 3d edit. Bailliere Tidall, London 
- Kumar, H.; Singh, B. D.; Bal. M. S.; Kamal Preetkaur.; Randhir singh; Sidhu, P. K. and Sandhu, K. S. (2007): Pathological and epidemiological investigations into listerial encephalitis in sheep. Small Rum. Research. 7 1: 293-297

- Ligios, C.; Viglietti, A.; Carta, P.J.; Dexter, G.; Agrimi, U. and Simmons, M.M. (2004): Clinicopathological findings in sheep from Sardinia showing neurological signs of disease. Vet. Rec. 154, 365370.

- Low J.C (2000): Listeriosis in Martine W.B. Aitken, I,D (Eds). Diseased of sheep 311(1 ed Blackwell oxford, pp 224-227.

- Low J. C., Renton C.P. (1985): Septicemia, encephalitis and abortion in a housed flock of sheep caused Listeria monocytogene type 1/2a. Vet. Rec. 1161 47-1 50.

- McClain,D.and Lee., W.H.(1988): Isolation o Listeria monocytogenes from meat and poultry. J. Assoc. Anal. Chem.(72): 660-664.

- Morin, D. E. (2004): Brain stem and cranial nerve abnormalities: Listeriosis, Otitis media, interno and pituitary abscess syndrome. Vet. Clin. Food Animal. 20: 243273

- Quinn, P.J., Carter, M.E., Markey, B., Carter, G.R., (1999): The streptococci and related cocci. In: Quinn, P.J., Ca'rter, M.E., Ivlarkey, B., Carter, G.R. (Eds.), Clinical Veterinary microbiology, Mosby International, Edinburgh, Scci: land, pp. 127-136.

- Roberts, A. J., and M. Wiedmann. (2003). Pathogen, host and environmental factors contributing to the pathogenesis of listeriosis. Cell. Mol. Life Sci, 60:904-918. 
- Schneider. D. J. (1994): Listeriosis In: Infectious Diseases of Live stock with special reference to South Africa. A text book Oxford Unvi, Press volume 11: 1374- 1377

- Scott P.R. (1993): A field study of ovine listerial meningoencephalitis with particular reference to cerebrospinal fluid analysis as an aid to diagnosis and prognosis. Br VetJ149:165-170

- Scott P.R. (2004): Diagnostic techniques and clinicopathoiogic findings in ruminant neurological disease. Vet. Clin. food animal, practice 20:215-230.

- Thanna (2002): Molecular grouping of Listeria monocytogenes based on the surface antigens in Listeria monocytogenes of serotype $4 \mathrm{~b}$. J. Bacteriol

- Van-Netten, P.: Perales, 1.Van Denoosdigk, Curtis. G.D.W. and Mossel, D.A.A.(1989): Liquid and solid selective differential media for the detection and enumeration of Listeria monocytogenes and other Listeria. Spp.Int J. Food. Microbiol. 8: 299-3 16. 